\title{
Ereignisse und ihre Wirkung auf die Themenagenda der Wählerschaft
}

\section{Evelyn Bytzek}

Der Einfluss von Ereignissen wird für eine Vielzabl an politischen Größen konstatiert, beispielsweise Wablergebnisse oder politische Reformen. Die Voraussetzung für einen solch bedeutenden Einfluss ist jedoch, dass Ereignisse die Themenagenda der Wäblerschaft bestimmen und so Druck auf die Politik ausüben. Ob diese Voraussetzung erfüllt wird, ist bislang jedoch nicht über Fallstudien hinausgehend untersucht worden. Der vorliegende Beitrag widmet sich daber der Untersuchung des Einflusses von Ereignissen auf die Themenagenda der deutschen Wählerschaft für den Zeitraum von 1991 bis 2003. Es kann gezeigt werden, dass Ereignisse die Themenagenda beeinflussen und sogar Themen, über die die Bürger eigene Erfabrungen haben, in den Hintergrund drängen können. Dieser Effekt ist jedoch äußerst kurz.

Schlagwörter: Agenda Setting, Zeitreihen, Längsschnittanalyse, Medienagenda, politische Agenda, Ereignisauswahl

\section{Einleitung}

Die Wirkung von Ereignissen auf unterschiedliche Bereiche der Politik ist nahezu unumstritten. So soll die Elbeflut die Bundestagswahl 2002 maßgeblich mitentschieden haben (Roth / Jung 2002: 7; Hartenstein / Müller-Hilmer 2002: 19). Ebenso wird der Erfolg der Grünen bei der Bundestagswahl 1987 auf umweltpolitische Ereignisse wie das Atomunglück in Tschernobyl und die Rheinverschmutzung aufgrund eines Brandes bei der Chemiefirma Sandoz in Basel zurückgeführt (Knochel / Lindgens 1990). Neben dem Einfluss von Ereignissen auf die Popularität und den Wahlerfolg unterschiedlicher Parteien wird ihnen ein weiterer wichtiger Effekt zugeschrieben: Sie stellen sog. Windows of Reform dar. Ereignisse gehen häufig mit Krisensituationen einher. Eine Krise liegt vor, wenn Unsicherheit über politische, gesellschaftliche und wirtschaftliche $\mathrm{Zu}-$ stände herrscht (vgl. Alink et al. 2001: 290). Ereignisse können eine solche Unsicherheit bewirken und sind somit häufig der Auslöser einer Krise. Nicht trotz, sondern gerade aufgrund ihrer grundsätzlichen Schädlichkeit beherbergen Krisen das Potenzial einer Reform des von der Krise betroffenen Bereiches, da durch die Krise der Status quo deutlich in Frage gestellt und die Notwendigkeit von Reformen offensichtlich wird. Krisen können daher zu Lerneffekten und somit zur Vermeidung von ähnlichen Begebenheiten in der Zukunft führen (vgl. Brändström et al. 2004: 192). Ähnliches stellt Birkland für sog. Focusing Events fest. Laut ihm haben Focusing Events eine große Wirkung auf die Policy-Tätigkeit der Regierung, da sie aufgrund ihrer Plötzlichkeit und Schädlichkeit nicht von der öffentlichen Agenda fernzuhalten sind. Hierdurch generieren sie einen öffentlichen Druck auf die Regierung, hinsichtlich des Ereignisses tätig zu werden, wenn sie vor der Wählerschaft gut dastehen will (Birkland 1997: 23f.). Birkland erweitert somit den Ansatz, Krisen und Ereignisse als Möglichkeit zur Reform zu betrachten, um die Dimension der Bürger / Wähler. Ihnen ist die Regierung letztlich verantwortlich, daher stellt das Wiederwahlmotiv den Antriebsgrund für Reformbemühungen dar. Die Voraussetzung hierfür ist jedoch, dass ein Ereignis nicht nur die Medienagenda, sondern auch die Themenagenda der Wählerschaft anführt. Denn eine 
Regierung kann nur für etwas zur Rechenschaft gezogen werden, das für die Wähler auch eine gewisse Wichtigkeit besitzt.

Der Zusammenhang zwischen Ereignissen und Themenagenda klingt trivial. Dennoch liegen praktisch keine über die Zeit gehenden Untersuchungen dazu vor, ob Ereignisse einen Einfluss auf die Themenagenda haben. Dies ist erstaunlich, da die Themenagenda der Wählerschaft für viele politische Größen eine Rolle spielt, wie beispielsweise für die oben angesprochenen Reformen oder auch für den Ausgang von Wahlen. Ereignisse können somit - vermittelt über die Themenagenda der Wählerschaft - einen nicht unwesentlichen Einfluss auf das politische Geschehen haben, so dass die fehlende empirische Überprüfung des Zusammenhangs zwischen Ereignissen und Themenagenda der Wählerschaft eine gravierende Forschungslücke darstellt. Die vorliegende Untersuchung widmet sich daher der Frage, ob Ereignisse eine Wirkung auf die Themenprioritäten der Wählerschaft haben. Als theoretischer Rahmen für die Untersuchung dieser Frage bietet sich der Agenda Setting-Ansatz an, wie in Abschnitt 2 ausführlich dargelegt wird. Hierfür wird zunächst aufgezeigt, wie die Themenagenda der Wählerschaft in „Normalzeiten“ und bei Ereignissen zustande kommt. Im Rahmen dessen wird ebenfalls der Frage nachgegangen, warum Ereignisse eine Wirkung auf die Themenagenda haben sollten und wie diese Wirkung aussieht. Da sich Untersuchungen im Rahmen des Agenda Setting jedoch in erster Linie mit Issues, also politischen Sachfragen oder Themen, und nicht mit Ereignissen beschäftigen, scheint es schon an dieser Stelle angebracht, kurz darauf einzugehen, was unter einem Ereignis verstanden wird. Obwohl, oder gerade weil dieser Begriff eine breite Anwendung erfährt, besteht wenig Konsens darüber, wie Ereignisse von Issues abzugrenzen sind (Shaw 1977: 20ff.). Grundlegend scheint bei Ereignissen zu sein, dass sie im Gegensatz zu Issues zeitlich und räumlich begrenzt sind (Kepplinger 2001: 119; Soroka 2002: 5f.). Wäre dies das einzige Charakteristikum von Ereignissen, müsste jedoch alles, was „passiert“, ein Ereignis sein. So wird der Begriff jedoch generell und auch hier nicht verstanden. Ereignisse geschehen überraschend, plötzlich und haben eine innovative, systemverändernde Kraft (Martin 2001: 209; Suter 2001: 181). Ereignisse können daher als (positive oder negative) Schocks angesehen werden und haben folglich eine gewisse Tragweite, beispielsweise für die Politik.

Über diese grundlegende Charakterisierung von Ereignissen hinaus ist die weitere Ausgestaltung des Ereignis-Begriffs an die jeweilige Forschungsfrage gebunden, d. h. der Ereignis-Begriff muss zur Identifikation von für die vorliegende Untersuchung relevanten Ereignissen „operationalisiert“ werden. Dies geschieht im dritten Abschnitt, in dem Kriterien aufgestellt werden, anhand derer potenziell wirksame Ereignisse ausgewählt werden können. Im Anschluss daran wird die auf diesen Kriterien basierende Ereignisauswahl vorgestellt. Nachdem der vierte Abschnitt das Vorgehen für die empirische Überprüfung der konstatierten Ereigniswirkung aufzeigt, wird diese Überprüfung in Abschnitt 5 anhand von Zeitreihenanalysen für Gesamtdeutschland und damit den Zeitraum von 1991 (da erst ab 1991 die benötigten Daten für Ostdeutschland vorliegen) bis 2003 vorgenommen.

\section{Die Themenagenda der Wählerschaft und Ereignisse}

Wie kommt die Themenagenda der Wähler zustande und welche Rolle spielen Ereignisse hierbei? Die Themenagenda der Wählerschaft wird in erster Linie von zwei Quellen gespeist: den eigenen Erfahrungen der Wähler, also realen Entwicklungen, und der Medienagenda. Ein Beispiel für Ersteres ist die wirtschaftliche Lage eines Landes und 
hierbei insbesondere die Entwicklung der Arbeitslosigkeit, die die Menschen direkt betrifft und daher das Potenzial hat, ohne Umweg über die Medienagenda auf die Agenda der Wählerschaft zu wirken (Soroka 2002: 23). Die Medienagenda wird hingegen dann besonders wichtig für die Agenda der Wählerschaft, wenn ein Thema für die Wähler nur schwer direkt erfahrbar ist. Dies trifft auf viele politische Themen zu, so dass die Wähler über einen Großteil der Politik in erster Linie durch die Medien unterrichtet werden. Die Unterscheidung zwischen Themen, die die Wähler direkt erfahren, und solchen, bei denen dies nicht möglich ist, wird auch in der Agenda Setting-Forschung vorgenommen. Erstere werden als obtrusive, letztere als unobtrusive Issues bezeichnet. Im Großen und Ganzen lässt sich zeigen, dass der Einfluss von direkt erfahrbaren Themen geringer ist als von Themen, die ausschließlich über die Medien erfahren werden können (Zucker 1978; McCombs 2004: 60ff.).

Die Medien wiederum sind bei politischen Themen auf Informationen über derzeit relevante Themen von politischen Akteuren angewiesen. Die Medienagenda wird daher wesentlich von der politischen Agenda beeinflusst. Insbesondere Regierungsakteuren und anderer politischer Prominenz wird zugetraut, über unterschiedliche Wege - von der allgemeinen Presseerklärung bis zum persönlichen Kontakt mit Journalisten - ihre Themen in der Medienberichterstattung unterzubringen oder die Vermeidung unliebsamer Themen veranlassen zu können (Berkowitz 1992; Edwards / Wood 1999). Neben Unterschieden in den thematischen Schwerpunkten zwischen Parteien und Politikern beeinflussen reale Entwicklungen die politische Agenda (Soroka 2002: 11). Zusammenfassend lässt sich daher sagen, dass in „Normalzeiten“, also wenn kein die Medienagenda beherrschendes Ereignis vorliegt, die Agenda der Wählerschaft im Wesentlichen von realen Entwicklungen und der Medienagenda beeinflusst wird, wobei die Medienagenda wiederum stark von der politischen Agenda abhängt. Dieser Zusammenhang wird in Abbildung 1 veranschaulicht.

\section{Abbildung 1: Zustandekommen der Themenagenda der Wäblerschaft in "Normalzeiten"}

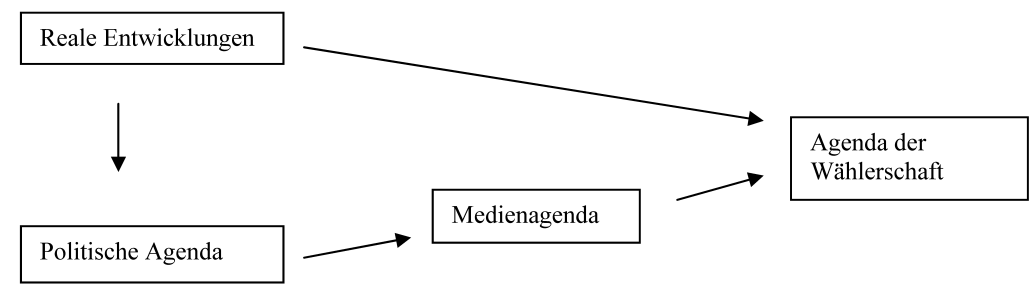

Wie jedoch wirken die drei Agenden zusammen, wenn ein Ereignis auftritt? Setzen Ereignisse, die häufig mit Krisen einhergehen, den oben aufgezeigten Prozess außer Kraft? Die in der Einleitung genannte Diskussion über Focusing Events gibt einen ersten Hinweis auf die besondere Wirkungsweise von Ereignissen: Sie sind weder von der Medienagenda noch von der Agenda der Wählerschaft fernzuhalten (Birkland 1997: 23f.). Hierdurch gewinnen Ereignisse das oben angesprochene Druckpotenzial auf die Politik. Ereignisse scheinen daher die Eigenschaft zu besitzen, die Medienagenda ohne den Umweg über die politische Agenda bestimmen zu können. Da sie für die wenigsten Personen direkt erfahrbar sind, ähneln sie den oben angesprochenen unobtrusive Issues (Zucker 1978) oder sog. Sensations-Issues, die ebenfalls nicht direkt erfahrbar, aber dennoch (be-)greifbar, also nicht abstrakt sind. Tatsächlich werden Sensations-Issues oft von Ereignissen ausgelöst (Soroka 2002: 25f.). Hierbei formt die Medienagenda sowohl 
die öffentliche als auch die politische Agenda. Das Zustandekommen der Agenda der Wählerschaft unterscheidet sich bei einem Ereignis wesentlich von dem in Abbildung 1 dargestellten Prozess: Die politische Agenda steht nicht am Beginn der Themensetzung, sondern an deren Ende, so dass politische Akteure das Ereignis nicht von der Agenda der Wählerschaft fernhalten können. Dieser Zusammenhang wird in Abbildung 2 nochmals aufgezeigt.

\section{Abbildung 2: Zustandekommen der Themenagenda der Wäblerschaft wäbrend eines Ereignisses}

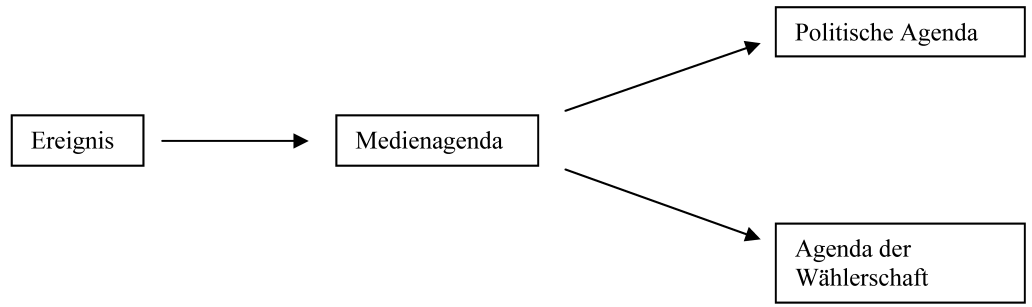

Doch warum haben Ereignisse diesen „durchschlagenden“ Effekt auf die Medienagenda? Der Grund hierfür ist die Newsworthiness von Ereignissen. Diese lässt sich auf viele Charakteristika von Ereignissen zurückführen, beispielsweise ihr plötzliches Eintreten, ihr Bezug zu Themen des Human Interest, oft auch ihre Schädlichkeit. Dies bewirkt, dass die Medien um Ereignisse „nicht herumkommen“, sie also berichten müssen (und auch wollen, da sie hierdurch Zuschauerzahlen / Auflagen steigern können). Der Weg von Ereignissen auf die Medienagenda geschieht also „automatisch“. Die Besonderheit von Ereignissen liegt folglich darin, dass sie die Medienagenda direkt, ohne Umweg über die Politik wie bei anderen politischen Themen, betreffen und von dort aus gleichzeitig auf die Agenda der Wählerschaft und die politische Agenda wirken. Im Gegensatz zu dieser Besonderheit ändert sich die grundlegende Beziehung zwischen Medienagenda und Agenda der Wählerschaft jedoch nicht: Die Medienagenda beeinflusst die Agenda der Wählerschaft, es sind also „gewöhnliche“ Agenda Setting-Effekte zu erwarten.

Trotz der Breite der Agenda Setting-Forschung ist der Einfluss der Medienagenda auf die Agenda der Wählerschaft generell wenig umstritten (für einen Überblick Brosius 1994; Dearing / Rogers 1996: 49ff.; McCombs 2004). Bisherige Ergebnisse beziehen sich jedoch in erster Linie auf Issues, also politische Themen. Ereignisse finden bislang hingegen wenig Beachtung. Dies ist wahrscheinlich weniger deswegen der Fall, weil die Wirkung der Medienagenda auf die Agenda der Wählerschaft als selbstverständlich angenommen wird. Vielmehr dürfte der Ursprung der Agenda Setting-Forschung als Medienwirkungsforschung in Wahlkämpfen, in denen politische Akteure die Wähler von „ihren“ Themen überzeugen müssen, für diese Lücke verantwortlich sein, auch wenn sich die Agenda Setting-Forschung schon lange nicht mehr auf diesen kurzen Zeitraum beschränkt (vgl. Williams Jr. / Larsen 1977). Die fehlende direkte Untersuchung von Ereigniswirkungen führt dazu, dass Ereignisse in der Regel unter Issues subsumiert, also nicht explizit ausgewiesen werden. Dies hat in manchen Fällen paradoxerweise zur Folge, dass der Einfluss eines so großen Ereignisses wie des Reaktorunfalls in Tschernobyl im April 1986 nicht als solcher benannt wird (Brosius / Kepplinger 1990). Auch wenn Issues im Vordergrund stehen, konnte durch diese Untersuchungen neben dem überwältigenden Einfluss der Tschernobyl-Katastrophe ganz generell gezeigt werden, dass die Berichterstattung zu Ereignissen die wahrgenommene Wichtigkeit des Themas, 
in dem sie angesiedelt sind, stärker zu steigern vermögen als die „normale“ Berichterstattung zu diesen Themen (MacKuen / Coombs 1981: Kap. 5; Wanta / Hu 1993; Berens 2001: Kap. 6; Krause / Fretwurst 2007). Trotz der Indirektheit dieses „Beweises“ zeigt sich also, dass Ereignisse eine große Wirkung auf die Medien- und von dort ausgehend auf die Agenda der Wählerschaft haben. Die direkte, über spezielle Issues hinausgehende Untersuchung von Ereigniswirkungen lohnt sich somit, da dies durch die Verbindung zwischen Issue und Ereignis einen wichtigen Baustein in der Agenda Setting-Forschung darstellt.

Was lässt sich vor dem Hintergrund bisheriger Befunde der Agenda Setting-Forschung hinsichtlich der Wirkung der Medien- auf die Agenda der Wählerschaft während eines Ereignisses erwarten? Wie oben schon gesagt, ähneln Ereignisse unobtrusive Issues, ihre Wirkung sollte daher sehr stark sein. Hinzukommt, dass neuartige und besonders eindringliche Stimuli, wie Ereignisse sie darstellen, viel Aufmerksamkeit bei den Rezipienten generieren (Reddy 1991: 112f.), was die Voraussetzung für eine starke Wirkung ist. So klar oben davon ausgegangen wurde, dass Ereignisse von der Medienagenda nur schwerlich fernzuhalten sind, so deutlich machen bisherige Befunde, dass ein Ereignis über die Medienagenda auch die Agenda der Wählerschaft beträchtlich beeinflussen sollte. Die Reaktionszeit der Agenda der Wählerschaft sollte darüber hinaus relativ kurz sein (McCombs 2004: 43), es wird folglich erwartet, dass bei Ereignissen so gut wie ohne Zeitverzögerung eine Wirkung der Medien- auf die Agenda der Wählerschaft beobachtet werden kann. Daneben deuten weitere Befunde der Agenda Setting-Forschung jedoch daraufhin, dass das Interesse sowohl der Medien- als auch der Agenda der Wählerschaft an einem Thema relativ schnell erlahmt (Downs 1972; Brosius 1994: 275) und darüber hinaus Agenda Setting-Effekte der Medien nur kurz anhalten (Iyengar / Kinder 1987: 30). Dies dürfte auch für die Wirkung von Ereignissen zutreffen, so dass die oben konstatierte und nicht unwesentliche Wirkung von Ereignissen auf die Medien- und Agenda der Wählerschaft dahingehend relativiert werden muss, als dass sie nur von kurzer Dauer sein dürfte.

Zusammenfassend lässt sich zum Zusammenhang zwischen Ereignissen und der Themenagenda der Wählerschaft sagen, dass eindeutig ein Effekt der Ereignisse, vermittelt über die Medienagenda, auf die Agenda der Wählerschaft zu erwarten ist und dass diese darüber hinaus andere Themen von der Agenda verdrängen sollten. Besonders stark ist der Effekt von Ereignissen dann einzustufen, wenn nicht nur andere über die Medien vermittelte, sondern auch direkt erfahrene Themen verdrängt werden. Dieser Effekt von Ereignissen sollte jedoch nur von kurzer Dauer sein. Konkret bedeutet dies, dass Ereignisse einerseits nur kurz auf der Medienagenda vertreten sein sollten und andererseits die Agenda der Wählerschaft kaum über den Zeitraum der Medienberichterstattung hinaus beeinflussen dürften. Bevor diese Erwartungen empirisch überprüft werden können, muss jedoch zunächst klargestellt werden, von welchen Ereignissen eine Wirkung auf die Agenda der Wählerschaft erwartet wird. Diese Ereignisse werden dann zur Überprüfung der genannten Erwartungen herangezogen. Der Auswahl relevanter Ereignisse widmet sich der nächste Abschnitt.

\section{Auswahlkriterien und Ereignisauswahl 1991-2003}

Trotz oder gerade wegen des weitläufigen Gebrauchs des Begriffs Ereignis ist eine saubere Trennung zwischen Ereignis und Issue schwierig, wie auch die Subsumierung von Ereignissen unter Issues in bisherigen Studien zeigt. Neben der allgemeinen Charakterisierung von Ereignissen als zeitlich und räumlich begrenzte, plötzlich auftretende 
Geschehnisse werden daher für die vorliegende Untersuchung spezifische Kriterien erarbeitet, anhand derer Ereignisse im nächsten Schritt ausgewählt werden können. Da die Untersuchung sich mit der Wirkung von Ereignissen auf die Agenda der Wählerschaft über die Medienagenda beschäftigt, ist die potenzielle Wirksamkeit eines Ereignisses im Rahmen des in Abbildung 2 aufgezeigten Zustandekommens der Agenda der Wählerschaft wesentlich für die Ereigniswahl. Dies trifft nicht auf alle Geschehnisse zu, die landläufig als Ereignis bezeichnet werden. Die Wirksamkeit eines Ereignisses kann durch die Anwendung der drei folgenden Kriterien sichergestellt werden: die Exogenität eines Ereignisses, sein Bezug zur Politik und eine große Medienpräsenz.

Ein Ereignis ist exogen, wenn es nicht von Politikern oder Parteien in Szene gesetzt wurde, wie beispielsweise Reden und (Auslands-)Reisen. Exogene Ereignisse treffen also „von außen“ auf die Politik. Andernfalls müssen Ereignisse als Thematisierungsbemühungen der Politik gelten. Daher werden von der Politik inszenierte Ereignisse auch als Pseudo-Ereignisse bezeichnet und somit von „echten“ Ereignissen abgegrenzt (Kepplinger 1992: 52). Diese Art von Ereignis bildet aber nicht den Gegenstand der vorliegenden Untersuchung, sondern würde dem in Abbildung 1 dargestellten Schema des Zustandekommens der Agenda der Wählerschaft entsprechen. Die vorliegende Untersuchung hat folglich die Wirkung sog. genuiner Ereignisse zum Gegenstand (Kepplinger 2001: 126).

Neben der Exogenität muss ein Ereignis einen Bezug zur Politik aufweisen, da hier aufgrund des theoretischen Rahmens die Agenda der Wählerschaft in Hinblick auf die Politik im Vordergrund steht. Hat ein Ereignis keinen Bezug zur Politik, wäre es sinnlos anzunehmen, dass das Ereignis einen Einfluss auf politische Größen im Allgemeinen und die Agenda der Wählerschaft im Speziellen hätte. Wie kann man nun einen Bezug zwischen Ereignis und Politik herstellen? Die Verursachung eines Ereignisses durch politische oder ihnen unterstellte administrative Akteure stellt den einfachsten Weg dar, greift aber für die vorliegende Arbeit zu kurz. Dies kann am Beispiel einer Naturkatastrophe veranschaulicht werden: Auch wenn eine direkte Verursachung eines Hochwassers o. Ä. durch politische Akteure nicht gegeben ist, kann ein solches Ereignis eine große Wirkung auf die Agenda der Wählerschaft haben, wie die Elbeflut 2002 beweist. Darüber hinaus ist nur schwer festzustellen, wer ein Ereignis verursacht hat. Aus diesen Gründen scheidet die Verursachung durch politische Akteure als Bezug zwischen Ereignissen und Politik aus.

Stattdessen werden die Erwartungen der Wählerschaft an die Politik herangezogen (siehe zu einem ähnlichen Konzept für den US-amerikanischen Fall: Ostrom / Simon 1985). Dies ist konsistent mit dem in der Einleitung beschriebenen Gedankengebäude, dass durch Ereignisse ein Druck auf politische Akteure generiert wird. Die Bürger scheinen beim Auftreten eines Ereignisses also zu erwarten, dass die Politik etwas dagegen tut. Dies ist einer der Gründe dafür, dass Ereignisse so stark auf die Agenda der Wählerschaft wirken. Nun stellt sich jedoch die Frage, welche Erwartungen die Bürger an die Politik haben. Was muss die Politik also in den Augen der Bürger unbedingt leisten? Die Erwartungen an die Politik werden sich zwischen den Bürgern leicht unterscheiden und auch von ihren politischen Einstellungen abhängen. Der Kern an Erwartungen an die Politik lässt sich jedoch aus den Staatsaufgaben ableiten. Auch wenn diese nur einen eng umrissenen Bereich betreffen und viele Bürger zusätzliche Erwartungen an die Politik haben werden, stellen sie rechtlich verbriefte Aufgaben des Staates und damit den Kernbestand der Erwartungen an die Politik dar. Zu den Staatsaufgaben gehören die friedliche Regelung der Beziehungen zu anderen Staaten in politischer und wirtschaftlicher Hinsicht und die Abwehr äußerer Feinde im außenpolitischen Bereich. 
Die innenpolitischen Aufgaben sind vielfältiger, zu ihnen gehören die Sicherung des Lebens, der Gesundheit und des Eigentums der Staatsbürger, die Aufrechterhaltung und Fortentwicklung der Rechts- und Wirtschaftsordnung, die Förderung der Wirtschaft, die Daseinsvorsorge in der Sozialordnung und die Förderung der Kultur (Reineck, 2003: 19f.; Avenarius, 2002: 25; Herzog, 1988). Diese staatlichen Aufgaben kann man zusammenfassen zu den drei Bereichen: Erhaltung des internationalen Friedens und der äußeren Sicherheit, Aufrechterhaltung der inneren Ordnung und Sicherheit von Leben und Besitz und Förderung der Wohlfahrt und der sozialen Sicherheit. Zusätzlich lässt sich auch der Schutz der natürlichen Lebensgrundlagen als Aufgabe des Staates nennen, der 1994 in das Grundgesetz, Artikel 20a, eingefügt und 2002 um den Tierschutz erweitert wurde. Umweltschutz im weiteren Sinne ist daher eine weitere staatliche Aufgabe (vgl. Kirchgäßner, 1996: 453f.). Darüber hinaus dürfte es unstrittig sein, dass die Bürger von der Politik erwarten, diese Aufgaben zu erfüllen und dabei redlich zu sein. Eine gute Verhaltensweise der Politiker zählt somit auch zu den Erwartungen der Bürger.

\section{Tabelle 1: Ereignisarten nach Bereichen staatlicher Aufgaben}

\begin{tabular}{|c|c|c|c|c|}
\hline $\begin{array}{l}\text { Frieden und } \\
\text { äußere Sicher- } \\
\text { heit }\end{array}$ & $\begin{array}{l}\text { Innere Ordnung } \\
\text { und Sicherheit von } \\
\text { Leben und Besitz }\end{array}$ & $\begin{array}{l}\text { Wohlfahrt und } \\
\text { soziale Sicherheit }\end{array}$ & Umweltschutz & $\begin{array}{l}\text { Redlichkeit der } \\
\text { Politik }\end{array}$ \\
\hline $\begin{array}{l}\text { Kriege } \\
\text { Friedens- } \\
\text { schlüsse } \\
\text { Akute Bedro- } \\
\text { hungen des } \\
\text { Weltfriedens } \\
\text { Abwendung } \\
\text { der akuten } \\
\text { Bedrohung }\end{array}$ & $\begin{array}{l}\text { Terroranschläge } \\
\text { Festnahme von } \\
\text { Terroristen } \\
\text { Krawalle und De- } \\
\text { monstrationen mit } \\
\text { Ausschreitungen } \\
\text { Außergewöhnliche } \\
\text { oder politisch moti- } \\
\text { vierte Straftaten } \\
\text { Unglücksfälle mit } \\
\text { einer hohen Zahl an } \\
\text { Opfern } \\
\text { Naturkatastrophen } \\
\text { Lebensmittelskan- } \\
\text { dale }\end{array}$ & $\begin{array}{l}\text { Außergewöhnli- } \\
\text { che Weltmarktge- } \\
\text { schehnisse } \\
\text { (Drohende) } \\
\text { Konkurse großer } \\
\text { deutscher Firmen } \\
\text { Demonstratio- } \\
\text { nen zur sozialen } \\
\text { Sicherheit }\end{array}$ & $\begin{array}{l}\text { Zwischen- und } \\
\text { Unfälle in } \\
\text { Kernkraftwer- } \\
\text { ken und weitere } \\
\text { Umweltskandale } \\
\text { friedliche } \\
\text { Großdemonstra- } \\
\text { tionen gegen die } \\
\text { Nutzung von } \\
\text { Kernkraft oder } \\
\text { zu anderen um- } \\
\text { weltpolitischen } \\
\text { Themen } \\
\text { Spektakuläre } \\
\text { Aktionen von } \\
\text { Umweltorgani- } \\
\text { sationen }\end{array}$ & $\begin{array}{l}\text { Politische } \\
\text { Skandale }\end{array}$ \\
\hline
\end{tabular}

Ereignisse, die einen dieser Bereiche betreffen, liegen somit in der Verantwortlichkeit der Politik. Von ihr wird daher erwartet, dass Anstrengungen unternommen werden, diese Aufgaben zu erfüllen, also adäquat auf das Ereignis zu reagieren. Bei Ereignissen dieser Art muss jedoch beachtet werden, dass der deutsche Staat überhaupt in der Lage sein muss, Aktionen als Reaktion auf das Ereignis in Gang zu setzen. Räumlich weit entfernte Ereignisse dürften daher nur bei direkter deutscher Beteiligung eine Wirkung auf die Agenda der Wählerschaft haben. Aus diesem Grund ist beispielsweise der Irakkrieg 2003 nicht in der Ereignisauswahl enthalten, da die Diskussion um eine Beteiligung Deutschlands als politisches Thema zu betrachten ist und deutsche Politiker während des Kriegs, dem eigentlichen Ereignis, durch die fehlende Teilnahme wenig Handhabe über das Ereignis hatten. Auf Basis der oben genannten Erwartungen an die Politik wurden relevante Ereignisarten identifiziert, die Tabelle $1 \mathrm{zu}$ entnehmen sind. 
Über die Exogenität eines Ereignisses und den Bezug zur Politik hinaus ist es wesentlich, dass ein Ereignis eine große Medienpräsenz besitzt. Wie in Abbildung 2 deutlich wird, können Ereignisse nur über die Medienagenda vermittelt einen Einfluss auf die Themenagenda der Wählerschaft haben, da sie für die wenigsten Menschen direkt erfahrbar sind. Für die folgende empirische Überprüfung eines Ereigniseinflusses muss also sichergestellt werden, dass die Ereignisse eine hohe Priorität auf der Medienagenda haben. Dies bedeutet jedoch nicht, dass man jedem Ereignis, über das in den Medien sehr stark berichtet wird, einen Einfluss auf die Agenda der Wählerschaft unterstellen kann. Hierfür muss, wie oben gesagt, ein Ereignis exogen sein und einen Bezug zur Politik besitzen.

Aus diesem Grund erfolgte die Ereignisauswahl in zwei Stufen: zunächst wurden aus Jahreschroniken (Harenberg 1995-2004) Ereignisse ausgewählt, die in das in Tabelle 1 dargestellte Schema passen. Die Medienpräsenz dieser Ereignisse wurde im nächsten Schritt anhand von Titelseiten der FAZ und des SPIEGELs festgestellt. Beide Medien haben eine Meinungsführerposition im deutschen Mediensystem inne (Kepplinger u. a. 1986: 267, Weischenberg u. a., 2006: 359). Daher kann man davon ausgehen, dass sie erstens als Qualitätsmedien über wesentliche Ereignisse berichten werden. Zweitens werden andere Medien ihnen in Thema und Tenor der Berichterstattung folgen. Eine Mindestmedienpräsenz, die an der Intensität und Länge der Berichterstattung in der FAZ und dem Erscheinen auf der Titelseite des SPIEGELs gemessen wurde, war notwendig, um in die Ereignisauswahl aufgenommen zu werden. Da bislang keine Erfahrungen dazu vorliegen, wie hoch die Präsenz eines Ereignisses sein muss, damit es eine Wirkung zeitigt, ist das gewählte Vorgehen recht komplex und geht auf alle Eventualitäten ein. Zunächst wurde erfasst, an welchen Tagen und mit wie vielen Artikeln ein Ereignis auf den Titelseiten der FAZ präsent war. Auf Basis dieser Daten wurde hinsichtlich der Länge der Präsenz festgelegt, dass ein Ereignis wenigstens an vier aufeinanderfolgenden Tagen (wobei ein „Pausentag“ erlaubt ist) auf der Titelseite der FAZ vertreten sein muss. Hierdurch werden wirkungslose „Eintagsfliegen“ ausgeschlossen, gleichzeitig wird der Kurzlebigkeit von Ereignissen Rechnung getragen. Hiermit liegt jedoch nur die Mindestpräsenz fest. Erfüllt ein Ereignis dieses Kriterium, kann es in den darauffolgenden Tagen und Wochen auch weniger präsent sein. Um eine endlose zeitliche Ausdehnung zu verhindern, die wiederum die Intensität der Berichterstattung schwächt, endet der Berichtszeitraum, wenn ein Ereignis mit weniger als drei Artikeln pro Woche berichtet wird. Nachdem die Mindestpräsenz hinsichtlich Länge und Berichtszeitraum definiert ist, wurde daran anschließend festgelegt, wie hoch die Intensität der Berichterstattung innerhalb dieses Zeitraums zu sein hat. Dass eine gewisse Intensität vorliegt, ist insbesondere bei länger andauernden Ereignissen wichtig, da diese wenig Wirkung zeigen werden, wenn über sie innerhalb eines größeren Zeitraums nur sporadisch berichtet wird. Das Kriterium, das angelegt wurde, sieht vor, dass ein Ereignis 75 \% seiner möglichen Präsenzintensität realisiert. Diese mögliche Präsenzintensität wurde anhand der Zahl der Tage im Berichtszeitraum, an denen eine Berichterstattung über das Ereignis möglich war, festgelegt. Obwohl dieses Vorgehen schon außerordentlich komplex ist, wurde mit der notwendigen Präsenz auf einer SPIEGEL-Titelseite eine weitere Hürde eingeführt, die der Befürchtung Rechnung trägt, durch die FAZ eine einseitige Ereignisberichterstattung zu erfassen. Die Einführung dieses Kriteriums stellt letztlich eine Verschärfung dar, da der auf einer SPIEGEL-Titelseite zur Verfügung stehende Platz im Vergleich zur täglich erscheinenden FAZ stark eingeschränkt ist. Etliche Ereignisse, die eine Mindestpräsenz in der FAZ vorweisen, scheiterten somit an dieser Hürde, wie beispielsweise die Entdeckung verstrahlter Castor-Transporte im Mai 1998 oder der Tod 
von Jürgen W. Möllemann durch einen verunglückten Fallschirmsprung im Juni 2003. Dies ist jedoch im Sinne der vorliegenden Untersuchung, da angenommen wird, dass nur außerordentlich präsente Ereignisse eine Wirkung haben (genauer zum Vorgehen Bytzek 2007).

Tabelle 2 gibt einen Überblick über die ausgewählten Ereignisse. Um die Verbindung zu den Kriterien Exogenität und Bezug zur Politik deutlich zu machen, wird in der Spalte „Ereignistyp“ aufgeführt, welcher in Tabelle 1 verzeichneten Ereignisklasse ein Ereignis als zugehörig angesehen wird.

Tabelle 2: Ausgewählte Ereignisse 1991-2003

\begin{tabular}{|c|c|c|}
\hline Ereignis & Ereignistyp & $\begin{array}{l}\text { Zeitraum der Medien- } \\
\text { präsenz }\end{array}$ \\
\hline rechtsextreme Gewalt: Hoyerswerda & außergewöhnliche Straftat & 24.09.-07.10.91 \\
\hline Lafontaine-Affäre & politischer Skandal & 10.06.-13.06.92 \\
\hline rechte Gewalt: Rostock & außergewöhnliche Straftat & 25.08.-01.09.92 \\
\hline rechte Gewalt: Mölln & außergewöhnliche Straftat & 24.11.-02.12.92 \\
\hline Briefbogenaffäre Möllemann & politischer Skandal & 23.12.-31.12.92 \\
\hline Schubladen-Affäre & politischer Skandal & 02.03.-22.05.93 \\
\hline rechte Gewalt: Solingen & außergewöhnliche Straftat & 01.06.-08.06.93 \\
\hline Festnahme durch die GSG9 & politischer Skandal & 29.06.-14.07.93 \\
\hline Plutoniumschmuggel & Kernenergie-Zwischenfall & 15.08.-25.08.94 \\
\hline Übertragbarkeit von BSE & Lebensmittelskandal & 26.03.-04.04.96 \\
\hline Oderflut & Naturkatastrophe & 23.07.-06.08.97 \\
\hline Lafontaine-Rücktritt & politischer Skandal & 12.03.-22.03.99 \\
\hline Kosovokrieg & Krieg & 25.03.-02.06.99 \\
\hline CDU-Spendenaffäre & politischer Skandal & 29.11.99-24.02.00 \\
\hline erster deutscher BSE-Fall & Lebensmittelskandal & 25.11.-23.12.00 \\
\hline Skandal um linke Vergangenheit Fischers & politischer Skandal & 04.01.-28.02.01 \\
\hline Flugskandal Scharpings & politischer Skandal & 01.09.-11.09.01 \\
\hline Terroranschläge am 11.9. in den USA & terroristischer Anschlag & 12.09.-22.09.01 \\
\hline Afghanistankrieg & Krieg & 08.10.-17.11.01 \\
\hline Spendenaffäre NRW-SPD & politischer Skandal & 05.03.-18.04.02 \\
\hline Amoklauf in Erfurt & außergewöhnliche Straftat & 27.04.-04.05.02 \\
\hline Antisemitische Äußerungen Möllemanns & politischer Skandal & 23.05.-07.06.02 \\
\hline Elbeflut & Naturkatastrophe & 12.08.-06.09.02 \\
\hline
\end{tabular}


Wie der Zeitraum der Medienpräsenz zeigt, haben viele Ereignisse tatsächlich eine sehr kurze „Lebensspanne“ auf der Medienagenda. Dauert ein Ereignis jedoch länger und kommen daher immer neue Informationen über das Ereignis, schwächt sich das $\mathrm{Me}-$ dieninteresse nicht ab, wie die CDU-Spendenaffäre 1999 / 2000 zeigt. Lange über die eigentliche Dauer eines Ereignisses hinaus hält das Medieninteresse jedoch in keinem Fall an, wie der Kosovokrieg veranschaulicht: Hier ließ das Medieninteresse tatsächlich schon vor dem offiziellen Waffenstillstand am 10. Juni 1999 deutlich nach. Insgesamt bestätigt sich daher die Vermutung, dass die Medien Ereignissen nicht lange ihre Aufmerksamkeit schenken, weswegen auch nicht mit einem lange anhaltenden Effekt von Ereignissen auf die Agenda der Wählerschaft zu rechnen ist.

Die in Tabelle 2 vorgestellten Ereignisse bildeten die Grundlage der empirischen Überprüfung der Ereigniswirkung auf die Agenda der Wählerschaft, deren Untersuchungsplan Thema des nächsten Abschnitts ist.

\section{Untersuchungsplan}

Im Folgenden soll überprüft werden, welche Wirkung die ausgewählten, auf der Medienagenda prominent vertretenen Ereignisse auf die Themenagenda der Wählerschaft haben. Im Grunde geht es folglich darum, die Wirkung der Medienagenda auf die Themenagenda der Wählerschaft im Falle eines Ereignisses zu untersuchen. Da eine große Präsenz auf der Medienagenda jedoch ein Auswahlkriterium von Ereignissen war, wird im Folgenden häufig lediglich von Ereigniswirkungen auf die Themenagenda der Wählerschaft gesprochen. Die gewählte Methode ist die Zeitreihenregression. Die Datenbasis für die folgende Untersuchung bildeten monatliche Politbarometer-Umfragen der Forschungsgruppe-Wahlen e.V., Mannheim. ${ }^{1} \mathrm{Da}$ die gesamtdeutsche öffentliche Meinung untersucht werden sollte, war die Einschränkung auf den Zeitraum von 1991 bis 2003 notwendig, da erst ab 1991 vollständige Daten zu Ostdeutschland vorliegen. Fehlende Werte kommen daher nur durch die bis 1997 übliche „Sommerpause“ bei den Befragungen zustande. Der Anteil an fehlenden Werten liegt jedoch unter der kritischen Größe von 5 Prozent, so dass keine explizite Imputation durchgeführt wurde. Stattdessen wurde die ARIMA-Routine zur Berechnung der folgenden Zeitreihenregressionen genutzt, die sich des Kalman-Filters bedient. Hierdurch findet eine implizite Imputation der fehlenden Werte statt (Green u. a. 1999: 187).

Die abhängige Variable bildet der Anteil der Arbeitslosigkeit an der Frage nach dem wichtigsten (oder zweitwichtigsten) Problem (sog. Agenda-Frage) mit einer Ost-WestGewichtung. ${ }^{2}$ Die Wahl fiel aus zwei Gründen auf diese Variable: Zum einen ist das Erstellen einer Zeitreihe aus dem Anteil der 23 einzelnen Ereignisse an der Frage nach dem wichtigsten Problem mit etlichen Schwierigkeiten verbunden. Die Agenda-Frage

1 Für Westdeutschland wurde das kumulierte Politbarometer 1977-2003 verwendet, das beim Zentralarchiv für Empirische Sozialforschung der Universität zu Köln unter der Bezeichnung s2391 erhältlich ist. Diese Kumulation enthält Daten für Ostdeutschland lediglich von 1996 bis 1998, so dass auf Jahreskumulationen zurückgegriffen werden musste. Die Jahreskumulationen für Ostdeutschland tragen die Bezeichnungen s2114 (1991), s2287 (1992), s2390 (1993), s2559 (1994), s2777 (1995), s3262 (1999), s3426 (2000), s3555 (2001), s3850 (2002) und s4001 (2003).

2 Der genaue Fragentext nach dem wichtigsten Problem lautet: „Was ist Ihrer Meinung nach gegenwärtig das wichtigste Problem in Deutschland?“. Bei der Frage nach dem zweitwichtigsten Problem wurde wie folgt gefragt: „Und was ist ein weiteres wichtiges Problem?“. 
ist eine offene Frage, die eher auf die Erfassung von Themen als von Ereignissen abzielt. Daher ist die Codierung der Ereignisse sehr ungenau. Während für manche Ereignisse, beispielsweise den Amoklauf eines Schülers an einem Erfurter Gymnasium 2002, eine eigene Kategorie besteht, werden andere, weniger singuläre Ereignisse wie politische Skandale unter einer groben Kategorie zusammengefasst. Darüber hinaus entsteht der Eindruck, dass versucht wird, Ereignisse so lange in eine der bestehenden Kategorien einzuordnen, bis diese zu „prominent“ für dieses Vorgehen geworden sind und sich eine eigene Kategorie „verdient“ haben. Diese Vorgehensweisen machen es nahezu unmöglich, die Präsenz der 23 gewählten Ereignisse auf der Themenagenda genau zu bestimmen.

Mit der Arbeitslosigkeit liegt hingegen ein durchgängig mit einer eigenständigen Kategorie versehenes und auch überaus wichtiges Thema vor. Abbildung 3 zeigt sehr deutlich, dass die Arbeitslosigkeit zu keiner Zeit als unwichtig betrachtet werden kann und im Gegenteil häufig das wichtigste Thema in der Wählerschaft war. Über die praktischen Gründe hinaus bietet sich die Verwendung der Arbeitslosigkeit aus einem inhaltlichen Grund als abhängige Variable an: Wenn Ereignisse eine Wirkung auf die Themenagenda der Wählerschaft haben, verdrängen sie notwendigerweise andere Themen. Aufgrund der engen Beziehung zwischen Medien- und öffentlicher Agenda ist hinsichtlich anderer von den Medien vermittelter Themen daher in erster Linie wichtig, dass ein Thema in den Medien prominent berichtet wird und dort andere Themen verdrängt. Dies ist jedoch bei Themen, über die die Bürger direkte Erfahrungen haben, nicht derart stark der Fall. Die Arbeitslosigkeit stellt ein solches „reales“ Thema oder obtrusive Issue dar, wodurch ihre Verwendung als abhängige Variable eine härtere Prüfung der Wirkung von Ereignissen bedeutet: Haben Ereignisse eine negative Wirkung auf die Wichtigkeit der Arbeitslosigkeit für die Wählerschaft, vermögen sie sogar „reale“ und nicht nur medienvermittelte Themen von der Agenda der Wählerschaft zu verdrängen, was durch die Wirkung von Ereignissen auf die Medienagenda quasi vorgegeben ist.

\section{Abbildung 3: Reale Entwicklung der Arbeitslosigkeit und Wichtigkeit des Themas}

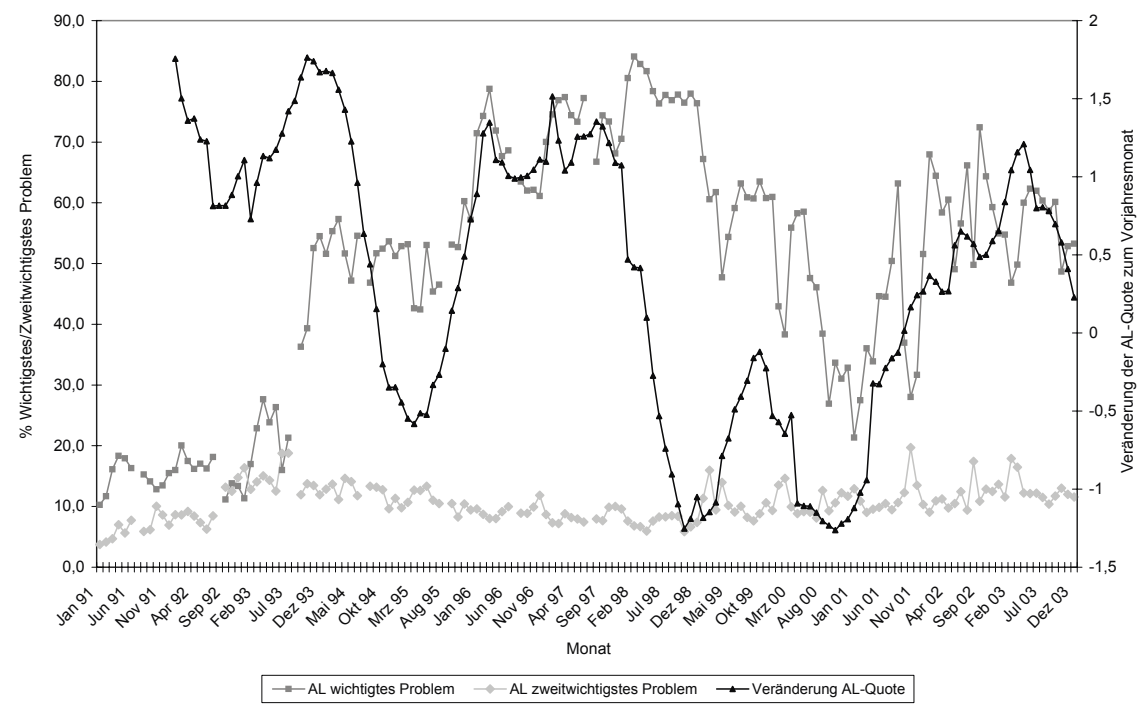


Ein weiterer Vorteil der Verwendung der Wichtigkeit der Arbeitslosigkeit als abhängige Variable ist, dass deren reale Entwicklung als unabhängige Variable in die folgenden Modelle einbezogen werden kann. Es wird erwartet, dass ein Anstieg der Arbeitslosigkeit auch zu einer Erhöhung des Anteils der Arbeitslosigkeit an der Frage nach dem wichtigsten Problem führt. Durch die Einbeziehung der Entwicklung der Arbeitslosigkeit wird erstens die Ereigniswirkung besser einschätzbar, da der Haupteinfluss auf die abhängige Variable im Modell enthalten ist. Zweitens wird so getestet, ob der in Abbildung 1 konstatierte Einfluss realer Entwicklungen auf die Themenagenda der Wählerschaft tatsächlich besteht. Bevor diese Behauptung anhand von Zeitreihenanalysen überprüft wird, zeigt Abbildung 3 den Zusammenhang zwischen dem Anteil der Arbeitslosigkeit an der Frage nach dem erst- und zweitwichtigsten Problem und der realen Entwicklung der Arbeitslosigkeit, gemessen als Veränderung der Arbeitslosenquote zum Vorjahresmonat, schon einmal grafisch auf.

Trotz der beträchtlichen Schwankungen in der Wichtigkeit der Arbeitslosigkeit für die Wählerschaft lässt sich grob ein Zusammenhang zwischen der realen Entwicklung der Arbeitslosigkeit und dem Anteil dieser am wichtigsten Problem erkennen. Ein Einfluss der realen Entwicklung auf die Themenagenda der Wählerschaft kann somit erwartet werden, zumindest hinsichtlich des wichtigsten Problems, da die Einstufung der Arbeitslosigkeit als zweitwichtigstes Problem deutlich konstanter ist und weniger stark von der realen Entwicklung abzuhängen scheint.

Die eigentlich interessierende unabhängige Variable bilden jedoch die 23 ausgewählten Ereignisse, die durch eine hohe Medienpräsenz als Auswahlkriterium die Medienagenda zu bestimmten Zeitpunkten widerspiegeln. Die Ereignisvariable ist eine Dummyvariable mit einer Eins, wenn ein Ereignis auftritt, und einer Null in ereignislosen Monaten. Auf Basis von Befunden der Agenda Setting-Forschung, die die Kurzlebigkeit dieses Effekts betonen, wird dann eine Wirkung unterstellt, wenn eine Befragung nicht länger als vier Wochen nach dem Ende der Medienpräsenz eines Ereignisses liegt.

\section{Abbildung 4: Ereignisse und die Wichtigkeit der Arbeitslosigkeit}

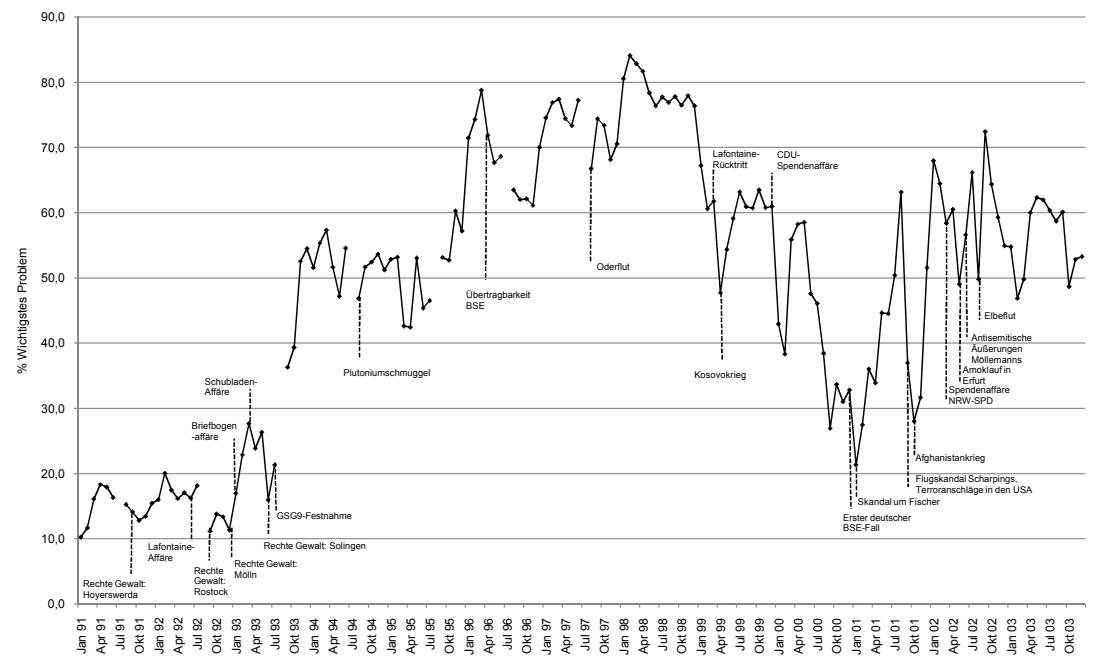


Es wird erwartet, dass Ereignisse eine negative Wirkung auf die Wichtigkeit der Arbeitslosigkeit haben, da sie diese teilweise von der Themenagenda der Wählerschaft verdrängen. Zunächst soll eine Abbildung einen ersten Hinweis auf den Zusammenhang zwischen Ereignissen und Themenagenda der Wählerschaft geben. In Abbildung 4 sind daher die Wichtigkeit der Arbeitslosigkeit (Problem 1) und das Auftreten der Ereignisse dargestellt (bei Ereignissen, von denen eine Wirkung bei mehreren Umfragezeiträumen erwartet wird, jeweils der erste Zeitraum).

Nachdem nun Datenbasis und Variablen vorgestellt wurden, widmet sich der nächste Abschnitt den Modellen, Berechnungen und Ergebnissen der vorliegenden Untersuchung.

\section{Modelle, Berechnungen und Ergebnisse}

Im Folgenden soll empirisch geklärt werden, ob Ereignisse, die eine prominente Position auf der Medienagenda einnehmen, einen Einfluss auf die Themenagenda der Wählerschaft haben, indem sie eine Verringerung der Wichtigkeit des vorherrschenden Themas, der Arbeitslosigkeit, bewirken können. Es geht also darum, Veränderungen zu erklären, nicht das Niveau der Wichtigkeit. Aus diesem Grund wird die abhängige Variable die Veränderungen der Wichtigkeit messen, also die Differenz der Wichtigkeit zum Vormonat. Die Veränderung der Wichtigkeit der Arbeitslosigkeit wird dann auf die Ereignisse und die reale Entwicklung der Arbeitslosigkeit zurückgeführt. Da die Veränderung der Arbeitslosenquote zum Vorjahresmonat keine stationäre Variable darstellt, muss auch sie differenziert werden und widerspiegelt daher einen groben Trend in der Entwicklung der Arbeitslosigkeit. Zusätzlich zu diesen beiden Variablen wird eine lagged dependent variable ( $\mathrm{t}-1)$ in das Modell einbezogen, um autoregressive Trends in der Wichtigkeit der Arbeitslosigkeit zu erfassen. Das Gesamtmodell sieht in Form einer Zeitreihenregressionsgleichung damit wie folgt aus:

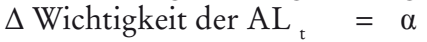

$$
\begin{aligned}
& +\beta_{1} \Delta \text { Wichtigkeit der AL }_{\mathrm{t}-1} \\
& +\beta_{2} \text { Ereignisse }_{\mathrm{t}} \\
& +\beta_{3} \Delta \text { Veränderung der AL zum Vorjahresmonat }_{\mathrm{t}-1} \\
& +\varepsilon_{\mathrm{t}}
\end{aligned}
$$

Es wird erwartet, dass Ereignisse einen negativen Effekt haben, die Arbeitslosigkeit als wichtigstes Problem also verdrängen, während die reale Entwicklung der Arbeitslosigkeit einen positiven Effekt haben sollte, mit steigender Arbeitslosigkeit also auch ihre Wichtigkeit auf der Themenagenda der Wählerschaft steigt. Die Ergebnisse dieses Modells für das wichtigste Problem sind in Tabelle 3 dargestellt. Wie deutlich zu sehen ist, hat die reale Entwicklung einen außerordentlich starken Effekt auf die Wichtigkeit der Arbeitslosigkeit. Dies ist als Bestätigung für die Behauptung zu werten, dass reale Entwicklungen eine wichtige Quelle für die Themenagenda darstellen, wie in Abbildung 1 konstatiert wurde.

Ereignisse haben in dem vorliegenden Modell entgegen der Erwartungen jedoch keinen signifikanten Effekt auf die Agenda der Wählerschaft. Dies könnte daran liegen, dass der konstatierte Wirkungszeitraum von bis zu vier Wochen schon zu lang für kurzlebige Ereignisse ist.

Aus diesem Grund wird die Ereignisvariable im folgenden Modell geteilt: Eine Variable erfasst Ereignisse, deren Medienpräsenz höchstens zwei Wochen vor einer Umfrage endet, sie werden als nahe Ereignisse bezeichnet. Eine zweite Variable enthält dagegen Ereignisse, bei denen erst drei oder vier Wochen nach Ende der Medienpräsenz 
eine Umfrage stattfand, sie werden als entfernte Ereignisse bezeichnet. ${ }^{3}$ In Form einer Regressionsgleichung sieht das Modell also wie folgt aus:

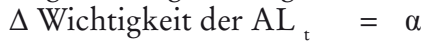

$$
\begin{aligned}
& +\beta_{1} \Delta \text { Wichtigkeit der AL }_{\mathrm{t}-1} \\
& +\beta_{2} \text { nahe Ereignisse }^{t} \\
& +\beta_{3} \text { entfernte Ereignisse }_{\mathrm{t}} \\
& +\beta_{4} \Delta \text { Veränderung der AL zum Vorjahresmonat }{ }_{\mathrm{t}-1} \\
& +\varepsilon_{\mathrm{t}}
\end{aligned}
$$

Es wird erwartet, dass die Aufteilung der Ereignisvariable dazu führt, dass nahe gelegene Ereignisse einen Effekt aufweisen, und zwar wie schon zuvor erwartet einen negativen Effekt. Von Umfragen weiter entfernt liegende Ereignisse sollten daher keinen signifikanten Effekt aufweisen und damit den fehlenden Einfluss der gesamten Ereignisvariable in Tabelle 3 erklären.

\section{Tabelle 3: Ereigniswirkungen anf die Wichtigkeit der Arbeitslosigkeit ${ }^{4}$}

\begin{tabular}{lc}
\hline & \begin{tabular}{c} 
Wirkung auf $\Delta$ Wichtigkeit Arbeitslosigkeit $^{(\text {Problem 1) }}{ }_{\mathrm{t}}$ \\
\hline$\Delta$ Wichtigkeit Arbeitslosigkeit (Problem 1) $_{\mathrm{t}-1}$
\end{tabular} \\
Reale Entwicklung Arbeitslosigkeit $_{\mathrm{t}-1}$ & $-0,10^{\mathrm{a}}(0,07)$ \\
Ereignisse $_{\mathrm{t}}$ & $9,23^{\mathrm{b}}(4,40)$ \\
\hline Konstante & $-2,11^{\mathrm{c}}(1,30)$ \\
\hline
\end{tabular}

$\mathrm{N}=124$, Standardfehler in Klammern, Signifikanzen: $a: \mathrm{p}<0,1 ; \mathrm{b}: \mathrm{p}<0,05 ; \mathrm{c}: \mathrm{p}<0,01$

Tabelle 4: Trennung der Ereignisvariablen in nabe und entfernte Ereignisse ${ }^{5}$

\begin{tabular}{lc}
\hline & ${\text { Wirkung auf } \Delta \text { Wichtigkeit Arbeitslosigkeit }}^{(\text {Problem 1) }}{ }_{\mathrm{t}}$ \\
\hline$\Delta$ Wichtigkeit Arbeitslosigkeit (Problem 1) & $-0,13^{\mathrm{a}}(0,07)$ \\
Reale Entwicklung Arbeitslosigkeit $_{\mathrm{t}-1}$ & $9,37^{\mathrm{b}}(4,30)$ \\
Nahe Ereignisse $_{\mathrm{t}}$ & $-5,22^{\mathrm{c}}(1,30)$ \\
Entfernte Ereignisse $_{\mathrm{t}}$ & $1,83(1,72)$ \\
\hline Konstante & $1,14(0,87)$ \\
\hline
\end{tabular}

$\mathrm{N}=124$, Standardfehler in Klammern, Signifikanzen: $\mathrm{a}: \mathrm{p}<0,1 ; \mathrm{b}: \mathrm{p}<0,05 ; \mathrm{c}: \mathrm{p}<0,01$

3 Durch dieses Vorgehen sind drei Ereignisse, die Briefbogenaffäre Möllemanns, die Oderflut und der Amoklauf in Erfurt, nur noch in der Auswahl der entfernten Ereignisse vorhanden, von denen keine Wirkung erwartet wird. Bei allen anderen Ereignissen wird lediglich der zweite oder dritte Wirkungszeitraum als entfernt betrachtet, sofern die entsprechende Umfrage nicht mehr als vier Wochen nach dem Ende der Medienpräsenz stattfand.

4 Alle verwendeten Variablen sind stationär. Das Modell ist weder durch Autokorrelation noch durch Heteroskedastizität gekennzeichnet. Für Teststatistiken siehe Anhang.

5 Alle verwendeten Variablen sind stationär. Das Modell ist weder durch Autokorrelation noch durch Heteroskedastizität gekennzeichnet. Für Teststatistiken siehe Anhang. 
Die Ergebnisse dieses Modells sind in Tabelle 4 verzeichnet. Die oben genannten Erwartungen werden von den empirischen Ergebnissen voll bestätigt: Nahe gelegene Ereignisse haben einen hochsignifikanten Effekt auf die Themenagenda der Wählerschaft, während entfernte Ereignisse keinen Einfluss mehr zu haben scheinen. Die Agenda Setting-Funktion der Medien bei einem Ereignis ist also extrem kurz und auf die Zeit der Berichterstattung und nur wenige Wochen danach beschränkt. Andererseits kann laut den Ergebnissen ein Ereignis die Wichtigkeit der Arbeitslosigkeit um durchschnittlich 5 Prozentpunkte senken. Bei länger andauernden Ereignissen besteht daher das Potenzial, die Arbeitslosigkeit als wichtigstes Thema zu verdrängen.

Bislang wurde nur der Anteil der Arbeitslosigkeit an der Frage nach dem wichtigsten Problem betrachtet, da diese im Untersuchungszeitraum sehr deutlich das wichtigste Thema darstellte (vgl. Abbildung 3). Die Themenagenda der Wählerschaft besteht jedoch nicht aus nur einem Thema. In den Umfragen wird dem Rechnung getragen, indem nach einem weiteren wichtigen Problem gefragt wird, das somit als zweitwichtigstes Thema betrachtet werden kann. Die Betrachtung des zweitwichtigsten Themas eröffnet die Möglichkeit, den Einfluss von Ereignissen auf die Themenagenda der Wählerschaft genauer zu untersuchen. Es stellt sich die Frage, ob Ereignisse nicht eher auf Platz 2 als auf Platz 1 landen, demnach also einen stärkeren Effekt auf die Frage nach dem zweitwichtigsten Problem hätten. Andererseits kann man anhand dieser Frage auch überprüfen, ob Ereignisse tatsächlich die Arbeitslosigkeit zum Teil auf den zweiten Platz verdrängen. Die Untersuchung dieser Fragen ist jedoch mit der Schwierigkeit verbunden, dass nicht nur die Arbeitslosigkeit, sondern etliche andere Themen als zweitwichtigstes Problem gelten können. So liegt die Arbeitslosigkeit nur bei etwa 10 Prozent der Nennungen (siehe Abbildung 3), dies aber recht konstant, so dass sie sich dennoch für die Überprüfung von Ereigniswirkungen anbietet. Ein Vorteil der Verwendung der Arbeitslosigkeit fällt jedoch weg: das Zurückführen ihrer Wichtigkeit auf die reale Entwicklung der Arbeitslosigkeit. Die relativ geringen Bewegungen deuten darauf hin, dass weniger die reale Entwicklung als vielmehr starke autoregressive Prozesse eine Rolle spielen (Abbildung 3). Daher wird statt der Veränderung der Arbeitslosenquote eine weitere lagged dependent variable ( $t-2)$ in das Modell integriert, um die autoregressiven Effekte zu kontrollieren. In Form einer Zeitreihenregressionsgleichung sieht das Modell also wie folgt aus:

$\Delta$ Wichtigkeit der AL $\mathrm{t}=\alpha$

$+\beta 1 \Delta$ Wichtigkeit der $\mathrm{AL}_{\mathrm{t}-1}$

$+\beta 2 \Delta$ Wichtigkeit der $\mathrm{AL}_{\mathrm{t}-2}$

$+\beta 3$ nahe Ereignisse

$+\beta 4$ entfernte Ereignisse ${ }_{t}$

$+\varepsilon_{+}$

Ein positiver Effekt der nahen Ereignisse wäre ein Indiz dafür, dass Ereignisse die Arbeitslosigkeit auf Platz 2 verdrängen. Ein negativer Effekt würde hingegen bedeuten, dass sie selbst eher als zweitwichtigstes Problem angesehen werden. Dies ist insbesondere für entfernt liegende Ereignisse wahrscheinlich, die im Zeitverlauf vom wichtigsten zum zweitwichtigsten Problem werden könnten. Die Ergebnisse dieses Modells sind Tabelle $5 \mathrm{zu}$ entnehmen.

Der positive Effekt der nahen Ereignisse bedeutet, dass der Anteil der Nennungen der Arbeitslosigkeit als zweitwichtigstes Problem steigt, wenn ein Ereignis auftritt. Dies deutet daraufhin, dass Ereignisse die Arbeitslosigkeit auf Platz 2 der Themenagenda der Wählerschaft verdrängen. Der Effekt ist jedoch sehr gering. Der nicht signifikante Effekt der entfernt liegenden Ereignisse kann bedeuten, dass Ereignisse im Zeitverlauf 
nicht von Platz 1 auf Platz 2 wandern, sondern plötzlich ganz von der Agenda verschwinden. Die Ergebnisse dieses Modells sind jedoch vorsichtig zu interpretieren, da die Arbeitslosigkeit nicht das einzige zweitwichtigste Problem darstellt und für den Einfluss anderer Probleme nicht kontrolliert werden kann.

\section{Tabelle 5: Auswirkung von Ereignissen auf das zweitwichtigste Problem ${ }^{6}$}

\begin{tabular}{lc}
\hline & \begin{tabular}{c} 
Wirkung auf $\Delta$ Wichtigkeit Arbeitslosigkeit $^{(\text {Problem 2) }}$ \\
\hline$\Delta$ Wichtigkeit Arbeitslosigkeit (Problem 2) $_{\mathrm{t}-1}$
\end{tabular} \\
$\Delta$ Wichtigkeit Arbeitslosigkeit (Problem 2) $_{\mathrm{t}-2}$ & $-0,45^{\mathrm{c}}(0,07)$ \\
Nahe Ereignisse $_{\mathrm{t}}$ & $-0,24^{\mathrm{c}}(0,08)$ \\
Entfernte Ereignisse $_{\mathrm{t}}$ & $0,73^{\mathrm{a}}(0,42)$ \\
\hline Konstante & $0,69(0,52)$ \\
\hline
\end{tabular}

$\mathrm{N}=125$, Standardfehler in Klammern, Signifikanzen: $a: p<0,1 ; b: p<0,05 ; \mathrm{c}: \mathrm{p}<0,01$

\section{Schlussfolgerung und Ausblick}

Die vorliegende Untersuchung ging der Frage nach, ob Ereignisse, vermittelt über die Medienagenda, einen Einfluss auf die Themenagenda der Wählerschaft haben. Diese Vermutung konnte bestätigt werden. Das Auftreten eines von den Medien prominent berichteten Ereignisses führt bei einem großen Teil der Bevölkerung dazu, dass andere Probleme, sogar solche, die selbst erfahren werden, auf Platz 2 der Agenda verdrängt werden. Insbesondere Ereignisse, die lange auf der Medienagenda vertreten sind, besitzen daher das Potenzial, die Themenagenda der Wählerschaft zu bestimmen. Diese „Macht“ von Ereignissen unterliegt aber deutlichen Grenzen: Der Effekt ist sehr kurz und hält nur wenig länger an, als ein Ereignis in den Medien präsent ist - und dies kann sehr kurz sein, wie Tabelle 2 zeigt.

Ereignisse haben in der Agenda Setting-Forschung, die sich vorrangig mit Issues beschäftigt, bislang wenig Beachtung gefunden. In der Konsequenz lagen lediglich Befunde dazu vor, dass Ereignisse die Wichtigkeit des Themas, dem sie angehören, stärker zu erhöhen vermögen als „normale“ Medienberichterstattung zu diesem Thema. Ereignisse wurden daher nur im Zusammenhang mit einem bestimmten Issue wahrgenommen und nicht als eigenständige Größen, die Einfluss auf die Medien-, politische und Themenagenda der Wählerschaft nehmen können.

Diese Forschungslücke wurde mit der vorliegenden Untersuchung geschlossen. Erstens konnte durch die Fokussierung auf Ereignisse deutlich gemacht werden, von welchen Ereignissen eine Wirkung auf die Themenagenda der Wählerschaft erwartet werden kann, während zuvor Ereignisse eher ad hoc in Erklärungen für eine plötzlich ansteigende Wichtigkeit eines Themas einbezogen wurden. Zweitens konnte deutlich gezeigt werden, dass auf der Medienagenda sehr präsente Ereignisse nicht nur manche Issues auf der Themenagenda nach vorne drängen, sondern darüber hinaus sogar direkt erfahrbare Themen wie die Arbeitslosigkeit in ihrer Wichtigkeit mindern können. Dies

6 Alle verwendeten Variablen sind stationär. Das Modell ist weder durch Autokorrelation noch durch Heteroskedastizität gekennzeichnet. Für Teststatistiken siehe Anhang. 
kann als Hinweis darauf verstanden werden, dass sich die Beziehungen zwischen politischer, Medien- und Agenda der Wählerschaft während eines Ereignisses im Vergleich zu Zeiten ohne große Ereignisse ändern und die Politik ihre Agenda Setting-Macht während eines Ereignisses weitgehend einbüßt. Der gezeigte Einfluss von über die Medien vermittelten Ereignissen auf die Themenagenda der Wählerschaft zeigt daher, dass die Kommunikation zwischen Politik, Medien und Bürgern nicht ausschließlich bestimmten Mustern folgt, sondern ebenso anfällig für externe Schocks ist.

Vordergründig scheint die Voraussetzung, dass Ereignisse als Windows of Reform dienen können, also gegeben zu sein, da sie tatsächlich Einfluss auf die Themenagenda der Wählerschaft nehmen. Dennoch bestehen massive Zweifel daran, dass Ereignisse tatsächlich die ihnen zugewiesene Rolle erfüllen: Die Kurzfristigkeit des Effekts zeigt, dass sich die Bürger schnell wieder anderen Themen zuwenden, lang andauernder Reformdruck also nur schwer aufgebaut werden kann. Und auch wenn für kurze Zeit der Druck auf die Politik besteht, Aktionen in Hinblick auf das Ereignis in Gang zu setzen, muss das nicht heißen, dass politische Reformen umgesetzt werden. Auf Ereignisse kann auch mit symbolischer Politik reagiert werden. Das bedeutet, dass der Zusammenhang zwischen Agenda der Wählerschaft und politischer Agenda zwar durch Ereignisse bestehen kann, die politische Agenda jedoch unterteilt sein kann in eine symbolische und Aktionsagenda (Pritchard 1992) und somit der kurzfristige öffentliche Druck auf der symbolischen Agenda abgebaut wird, notwendige grundlegende Reformen also nicht angegangen werden müssen, da die öffentliche Aufmerksamkeit für das Ereignis hierfür nicht lange genug anhält.

\section{Literatur}

Alink, Fleur / Boin, Arjen / 't Hart, Paul (2001): Institutional Crises and Reforms in Policy Sectors: The Case of Asylum Policy in Europe. In: Journal of European Public Policy, 8, 286-306.

Avenarius, Herrmann (2002): Die Rechtsordnung der Bundesrepublik Deutschland. Bonn: Bundeszentrale für politische Bildung.

Berens, Harald (2001): Prozesse der Thematisierung in publizistischen Konflikten. Ereignismanagement, Medienresonanz und Mobilisierung am Beispiel von Castor und Brent Spar. Wiesbaden: Westdeutscher Verlag.

Berkowitz, Dan (1992): Who Sets the Media Agenda? The Ability of Policymakers to Determine News Decisions. In: Kennamer, J. David (Hrsg.), Public Opinion, The Press, and Public Policy. Westport: Praeger, 81-102.

Birkland, Thomas A. (1997): After Disaster. Agenda Setting, Public Policy, and Focusing Events. Washington: Georgetown Press.

Brändström, Annika / Bynander, Fredrik / 't Hart, Paul (2004): Governing by Looking Back: Historical Analogies and Crisis Management. In: Public Administration, 82, 191-210.

Brosius, Hans-Bernd (1994): Agenda Setting nach einem Vierteljahrhundert Forschung: Methodischer und theoretischer Stillstand? In: Publizistik, 39, 269-288.

Brosius, Hans-Bernd / Kepplinger, Hans Mathias (1990): The Agenda Setting-Function of Television News. Static and Dynamic Views. In: Communication Research, 17, 183-211.

Bytzek, Evelyn (2007): Ereignisse und ihre Wirkung auf die Popularität von Regierungen, BadenBaden: Nomos.

Dearing, James W. / Rogers, Everett M. (1996): Agenda Setting. Thousand Oaks: Sage.

Downs, Anthony (1972): Up and Down with the Ecology - the „Issue-Attention Cycle“. In: Public Interest, 28, 38-50.

Edwards, George C. III / Wood, B. Dan (1999): Who Influences Whom? The President, Congress, and the Media. In: American Political Science Review, 93, 327-344. 
Green, Donald P. / Gerber, Alan S. / de Boef, Suzanna (1999): Tracking Opinion Over Time: A Method for Reducing Sampling Error. In: Public Opinion Quarterly, 63, 178-192.

Gujarati, Damodar (2003): Basic Econometrics. Boston u. a.: McGraw-Hill.

Harenberg, Bodo (Hrsg.) (1991-2004): Die Chronik-Bibliothek des Jahrhunderts. Tag für Tag in Wort und Bild (bis 2001: Die Chronik-Bibliothek des 20. Jahrhunderts), Band 1991-2003. Gütersloh u. a.: Chronik-Verlag.

Hartenstein, Wolfgang / Müller-Hilmer, Rita (2002): Die Bundestagswahl 2002: Neue Themen neue Allianzen. In: Aus Politik und Zeitgeschichte, B49-50, 18-26.

Herzog, Roman (1988): Ziele, Vorbehalte und Grenzen der Staatstätigkeit. In: Isensee, Josef / Kirchhof, Paul (Hrsg.), Handbuch des Staatsrechts der Bundesrepublik Deutschland, Band III: Das Handeln des Staates. Heidelberg: Müller, 83-120.

Iyengar, Shanto / Kinder, Donald R. (1987): News that Matters. Television and American Opinion. Chicago / London: Chicago University Press.

Kepplinger, Hans Mathias / Donsbach, Wolfgang / Brosius, Hans-Bernd / Staab, Joachim Friedrich (1986): Medientenor und Bevölkerungsmeinung. Eine empirische Studie zum Image Helmut Kohls. In: Kölner Zeitschrift für Soziologie und Sozialpsychologie, 38, 247-279.

Kepplinger, Hans Mathias (1992): Ereignismanagement. Wirklichkeit und Massenmedien. Zürich: Ed. Interfrom.

Kepplinger, Hans Mathias (2001): Der Ereignisbegriff in der Publizistikwissenschaft. In: Publizistik, 46, 117-139.

Kirchgäßner, Gebhard (1996): Umweltschutz als Staatsaufgabe. Einige Überlegungen aus ökonomischer Perspektive. In: Grimm, Dieter (Hrsg.), Staatsaufgaben. Baden-Baden: Nomos, 453485.

Knochel, Manfred / Lindgens, Monika (1990): Fünf-Prozent-Hürde und Medienbarriere. Die Grünen im Bundestagswahlkampf 1987: Politik, Medienpräsenz und Resonanz in der Wählerschaft. In: Kaase, Max / Klingemann, Hans-Dieter (Hrsg.), Wahlen und Wähler. Analysen aus Anlass der Bundestagswahl 1987. Opladen: Westdeutscher Verlag, 569-618.

Krause, Birgit / Fretwurst, Benjamin (2007): Kurzfristige Agenda Setting-Effekte von Fernsehnachrichten. Eine Zeitreihenanalyse am Beispiel Ausländerfeindlichkeit und Rechtsradikalismus. In: Krause, Birgit / Fretwurst, Benjamin / Vogelgesang, Jens (Hrsg.), Fortschritte der politischen Kommunikationsforschung. Festschrift für Lutz Erbring. Wiesbaden: VS Verlag für Sozialwissenschaften, 171-196.

MacKuen, Michael B. / Coombs, Steven L. (1981): More than News. Media Power in Public Affairs, Beverly Hills / London: Sage.

Martin, Jean-Clément (2001): Für eine Typologie der „Ereignisse“: Das Beispiel des Vendéekrieges. In: Suter, Andreas / Hettling, Manfred (Hrsg.), Struktur und Ereignis (Sonderheft 19, Geschichte und Gesellschaft. Zeitschrift für Historische Sozialwissenschaft). Göttingen: Vandenhoeck \& Ruprecht, 208-223.

McCombs, Maxwell E. (2004): Setting the Agenda. The Mass Media and Public Opinion. Cambridge: Polity Press.

Ostrom Jr., Charles W. / Simon, Dennis M. (1985): Promise and Performance: A Dynamic Model of Presidential Popularity. In: American Political Science Review, 79, 334-358.

Pritchard, David (1992): The News Media and Public Policy Agenda. In: Kennamer, J. David (Hrsg.), Public Opinion, The Press, and Public Policy. Westport: Praeger, 103-112.

Reddy, Peter (1991): Aufmerksamkeit und das Erlernen von Fertigkeiten. In: Gerstenmaier, Jochen (Hrsg.), Einführung in die Kognitionspsychologie. München u. a.: UTB Wissenschaft, 91-119.

Reineck, Karl-Michael (2003): Allgemeine Staatslehre und Deutsches Staatsrecht, Hamburg: Maximilian-Verlag.

Roth, Dieter / Jung, Matthias (2002): Ablösung der Regierung vertagt: Eine Analyse der Bundestagswahl 2002. In: Aus Politik und Zeitgeschichte, B49-50, 3-17.

Shaw, Donald L. (1977): The Press Agenda in a Community Setting. In: Shaw, Donald L. / McCombs Maxwell E. (Hrsg.), The Emergence of Political Issues: The Agenda Setting Function oft he Press, St. Paul u.a.: West Publishing Co., 19-31. 
Soroka, Stuart N. (2002): Agenda Setting Dynamics in Canada. Vancouver: UBC Press.

Suter, Andreas (2001): Ereignisse als strukturbrechende und strukturbildende Erfahrungs-, Entscheidungs- und Lernprozesse. Der schweizerische Bauernkrieg von 1653. In: Suter, Andreas / Hettling, Manfred (Hrsg.), Struktur und Ereignis (Sonderheft 19, Geschichte und Gesellschaft. Zeitschrift für Historische Sozialwissenschaft). Göttingen: Vandenhoeck \& Ruprecht, 175-207.

Wanta, Wayne / Hu, Yu-Hei (1993): The Agenda Setting Effects of International News Coverage: An Examination of Differing News Frames. In: International Journal of Public Opinion Research, 5, 250-263.

Weischenberg, Siegfried / Malik, Maja / Scholl, Armin (2006): Journalismus in Deutschland 2005. In: Media Perspektiven, 7, 346-361.

Williams Jr., Wenmouth / Larsen, David C. (1977): Agenda Setting in an Off-Election Year. In: Journalism Quarterly, 54, 744-749.

Zucker, Harold G. (1978): The Variable Nature of News Media Influence. In: Ruben, Brent D. (Hrsg.), Communication Yearbook, Vol. 2. New Brunswick: Transaction Books, 225-240.

\section{Anhang}

Tabelle A1: $\quad$ Ergebnisse der Augmented Dickey-Fuller-Tests anf Stationarität

\begin{tabular}{lccc}
\hline Zeitreihe & $\mathrm{Z}(\mathrm{t})$ & $\begin{array}{c}\text { Kritischer Wert bei } \\
\text { \%-Signifikanzniveau }\end{array}$ & $\begin{array}{c}\text { Zahl der } \\
\text { Beobachtungen }\end{array}$ \\
\hline$\Delta$ AL wichtigstes Problem (Problem 1) & $-12,378$ & $-3,499$ & 133 \\
$\Delta$ AL wichtigstes Problem (Problem 2) & $-16,439$ & $-3,499$ & 133 \\
$\Delta$ Veränderung der Arbeitslosenquote & $-8,236$ & $-3,496$ & 141 \\
zum Vorjahresmonat & $-7,028$ & $-3,492$ & 155 \\
Ereignisse (gesamt) & $-7,497$ & $-3,492$ & 155 \\
nahe Ereignisse & $-11,458$ & $-3,492$ & 155 \\
entfernte Ereignisse & & & \\
\hline
\end{tabular}

Tabelle A2: $\quad$ Ergebnisse der Tests auf Autokorrelation und Heteroskedastizität ${ }^{7}$

\begin{tabular}{lcc}
\hline Test auf & Autokorrelation & Heteroskedastizität \\
\hline kritischer Wert auf 1 \%-Niveau: & 7,88 & 10,60 \\
Modell 1 (Problem 1) & $-3,49$ & 2,13 \\
Modell 2 (Problem 1) & $-3,53$ & 6,01 \\
Modell 3 (Problem 2) & $-4,88$ & 9,31 \\
\hline
\end{tabular}

7 Für die Überprüfung auf Autokorrelation wurde Durbin's M-Test genutzt (Gujarati 2003: 473f.), für die Überprüfung auf Heteroskedastizität ein verkürzter White-Test (Gujarati 2003: 434). 